\title{
Young Students Exploring Cardinality by Constructing Infinite Processes
}

\author{
Ken Kahn ${ }^{1}$, Evgenia Sendova ${ }^{2}$, Ana Isabel Sacristán ${ }^{3}$, Richard Noss ${ }^{4}$ \\ ${ }^{1}$ London Knowledge Lab, Institute of Education, 23-29 Emerald Street, London \\ WC1N 3QS, United Kingdom, Phone: +44 (0)20 7763 2137, Fax: +44 (0)20 7763 \\ 2138,toontalk@gmail.com
}
${ }^{2}$ Institute of Mathematics and Informatics, Bulgarian Academy of Sciences, jenny@math.bas.bg

${ }^{3}$ Dept. of Mathematics Education, Cinvestav, asacrist@cinvestav.mx

${ }^{4}$ London Knowledge Lab, Institute of Education,r.noss@ioe.ac.uk

\begin{abstract}
In this paper, we describe the design and implementation of computer programming activities aimed at introducing young students (9-13 years old) to the idea of infinity, and in particular, to the cardinality of infinite sets. This research was part of the WebLabs project where students from several European countries explored topics in mathematics and science by building computational models and programs, which they shared and discussed. We focus on a subset of the work in which students explored concepts of cardinality of infinite sets by interpreting and constructing computer programs in ToonTalk, a programming language and environment that is especially well-suited for young students. Our hypothesis is that via carefully designed computational explorations within an appropriately constructed medium, infinity can be approached in a learnable way that does not sacrifice the rigour necessary for mathematical understanding of the concept, and at the same time contributes to introducing the real spirit of mathematics to the school classroom.
\end{abstract}

Keywords Infinity, cardinality, ToonTalk, constructionism, programming

\section{Introduction}

The infinite! No other question has ever moved so profoundly the spirit of men! These words of David Hilbert (quoted in Maor, 1987) have been a motivating challenge for a number of mathematics educators in their attempt to convey the real spirit of mathematics, by discussing deep ideas with students at school age.

In this paper we present our own attempt at introducing young students to this topic, through computational constructions that lead to the exploration of the cardinality of infinite sets. We were mindful that the infinite is particularly complex due to its contradictory nature. Tall (2001) has noted that the ideas that adolescents have of infinite concepts are influenced by their prior experiences; and since they tend to meet the notion of "limit" in some dynamic sense before the notion of one to one correspondences between infinite sets, this potential view of infinity clashes with the notion of infinite cardinals. He thus attempted to introduce a young 7-year old student to the concept of cardinal infinity by comparing infinite sets to see what this did to his intuitions; the results were mixed, since the child developed conflicting interpretations (interpreting infinity as a very large number - a result consistent with that of Falk and Ben-Lavy, 1989), but nevertheless showed a great versatility in thinking, producing some surprising insights of his own. Tall (2001) concluded that the young have "surprisingly sophisticated and complex ideas" of infinity "that deserve to be treated on their own terms with respect". It is also relevant for us that for comparing infinite quantities, Tsamir (2001) encouraged students to reflect on their thinking and to use the one-to-one correspondence criterion as the only valid method for comparing infinite quantities.

It is clear, however, that the intuitions that students have with respect to infinity are liable to change (Fischbein et al, 1979). In this sense, Monaghan (2001) found that the language and context that is used influences students' responses and conceptions; at the same time, he implicitly encourages activities that develop and change students' conceptions of infinity during the course of relevant activities. Sacristán (1997) carried out a study that attempted to do that (also reported in Sacristán \& Noss, 2008), where 13-14 year old students had the opportunity to explore and develop concepts of infinite processes and objects in a computational setting; in that case the 
available tools seemed to mediate students' understandings in rich ways, allowing them to discriminate subtle features of infinite processes, and coordinate hitherto unconnected or conflicting intuitions concerning infinity through what was there called 'representational moderation'. That study, like the current one, is about the possibilities of constructions and explorations, through computational infrastructures and representations, for the development of intuitions of infinity-related concepts at early ages.

Our intention here was to provide support to students to explore computationally and to discuss many of the major ideas of the cardinality of infinite sets. In what follows, we describe how students programmed non-terminating processes that produced infinite sequences including the natural numbers, the even numbers, the integers, and the rational numbers, while simultaneously constructing one-to-one correspondences between the respective sets. They shared and discussed their constructions with other students and reflected together on related questions posed by the researchers/teachers. Our hypothesis was that the curious learner could learn some deep and interesting mathematics without first having mastered the techniques that are normally only accessible to the few.

Thus, this paper explores the fundamental idea that the apparent difficulty of a concept, its appropriateness for a given age or 'ability' of student, is often contingent not so much on the concept itself, but on the way it is represented. This hypothesis looks like an instructional one, akin to Bruner's assertion (roughly) that any idea can be taught if it is taught appropriately. But this misses an essential epistemological point: that the re-representation of the concept can transform the knowledge to be taught. A new representation can consequently change how and to whom a concept is taught. diSessa (2000), for example, shows how the emergence of algebra in the seventeenth century democratised Newton's laws of motion - hitherto only expressible in extensive linguistic circumlocutions and geometrical detail - to a succinct and learnable set of three short equations. Similarly Wilensky and Papert use representation of numbers in Roman numerals to show just how sensitive simple (as we would see it now) arithmetic procedures are to the ways in which quantities are represented (Wilensky \& Papert 2010). Further, this example shows how the 'right' representations are unsurprisingly the ones that surface culturally.

Accordingly, this paper will set out some alternative representational systems (see Kaput, Noss, \& Hoyles 2002) for learning about infinity. Infinity is certainly a contender for the most challenging mathematical idea of the second millennium, and there is a case to be made that the last century or two were centrally focused on the idea. For this reason, if no other, there emerged an extensive algebraic machinery for dealing with the infinite, one that developed as a finely-tuned engine for thinking about the unthinkable. And yet this engine - a source of power to the few - became an obstacle to the many; even those - like most children - who have intuitions and curiosity concerning the infinite were simply unable to master the machinery sufficiently to express any of infinity's key ideas using it. This paper will show, on the contrary, that it is possible to design a representational system that makes the infinite accessible, one that affords a positive response to the inherent curiosity it provokes.

\section{Methodology and Design}

In an attempt to help students approach infinity-related ideas by providing them with an alternative formalism based on a computational setting, we designed a learning trajectory (Simon \& Tzur, 2004), that is a goal for students' learning, the tasks on which they should work, and hypotheses concerning students' learning processes.

The design of a relevant learning trajectory involved two challenges: (i) designing a path through the space of concepts related to the cardinality of infinite sets, and (ii) re-conceptualising the knowledge in ways that are enabled by computational explorations. We thus designed a series of activities, partially structured through worksheets, to lead the students to construct nonterminating processes in a specialised computer environment (ToonTalk) to produce the natural numbers, the even numbers, the integers, and the rational numbers.

We begin by introducing some facets of the ToonTalk environment that lend themselves particularly well as a basis for the exploration of infinite sequences, and more precisely, a framework or platform on which to design tools and activities for exploring infinity. 


\section{ToonTalk as an environment for exploring infinity}

In ToonTalk, programmers enter an animated world where each virtual element expresses an instance of a computational abstraction. For instance - as shown in detail in the example further below (Figure 1) - data structures are represented by boxes, communication and synchronisation mechanisms are represented by "birds", and "trucks" are used to express spawning of new processes. Users build programs by training "robots" to manipulate boxes with data, perform arithmetic calculations, give birds messages to deliver, and other actions; they train these robots using specific example data and then recover generality (Kahn, 2001).

Crucial for our studies was ToonTalk's support for concurrent computations and communication that enables the creation of multiple interacting infinite processes. ToonTalk robots are implicitly iterative - a robot executes an infinite loop unless being explicitly programmed to terminate. Expressing an infinite computation (such as generating the natural numbers) is possible in all programming languages. Many programming languages also implement the concept of threads or processes so that a computation can consist of more than one infinite computation. An important and unique feature of ToonTalk however, is that it supports communication and coordination between these infinite processes.

Consider the challenge of constructing a program to compute and visualise the natural numbers. In an ordinary programming language, this would be accomplished by a program such as the following:

\section{set $\mathrm{n}$ to 0 then \\ do forever set $n$ to $n+1$ and print $n$}

The difficulties arise when one needs multiple infinite sequences and the ability to perform operations upon those sequences. We are not aware of any child-accessible programming language other than ToonTalk that is capable of supporting computations on infinite sequences.

In order to construct a ToonTalk robot generating the natural numbers, it is sufficient to train it to repeatedly drop one number on another, when given a box with two inputs (0 and 1). As a result an animation is displayed of the robot copying the second number, dropping it on the first number. Then the numbers are smashed together to produce 1 (the sum of 0 and 1). The robot is then seen copying the other 1 , dropping it on top of the 1 in the first hole of the box to produce 2 , etc. Thus the consecutive natural numbers appear one at a time as a current content of a box (Figure 1).

(a)

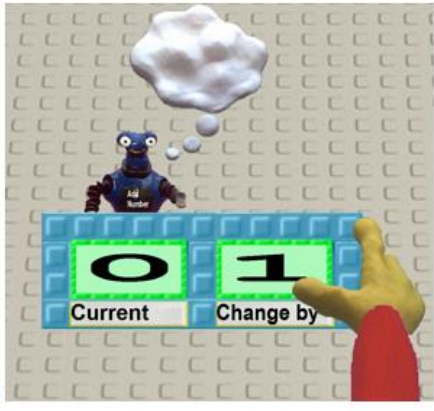

(c)

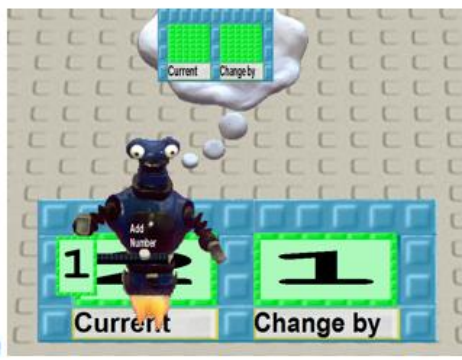

(b)

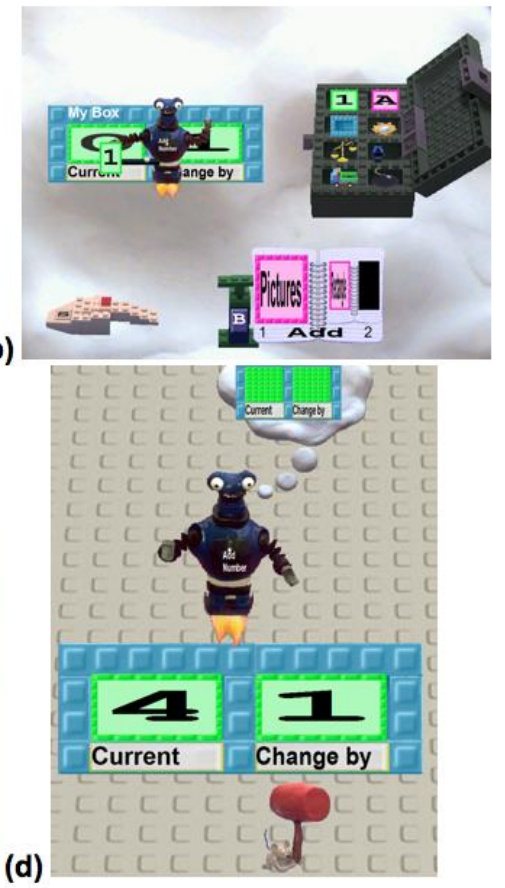

Figure 1 -Training a robot to produce an arithmetic progression: (a) Initiating the training with specific data; (b) Training the robot to copy the content of the second hole and adding it to the first 
hole; (c) The robot in the middle of the third iteration; (d) The robot at the end of its fourth iteration

Suppose now that one wants to compute both the natural numbers and the even numbers. One way to do this in ToonTalk is simply to copy the Add Number robot and change the content of the Change by box (Figure 2).

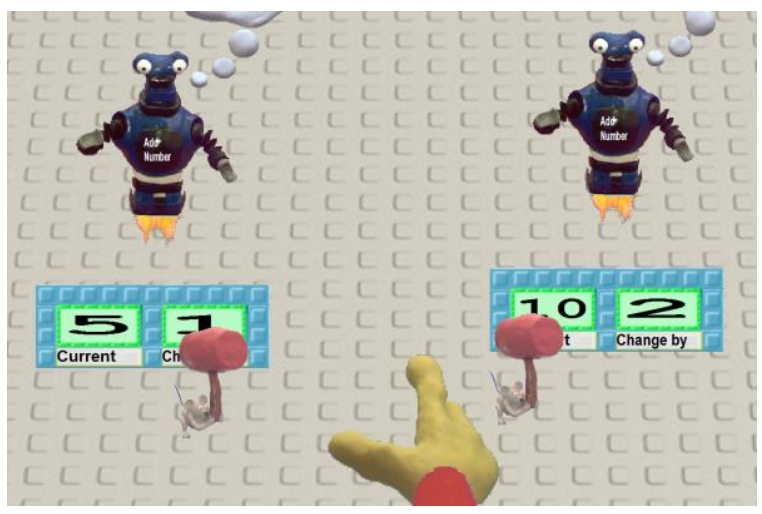

Figure 2 - Two copies of the Add Number robot running simultaneously with different inputs In a conventional program it is difficult to provide access to the state of one process to other processes. There are issues of how to reference the data and how to avoid conflicts. To perform our activities we want (i) to produce a computational object that acts like the sequence of natural numbers, and (ii) to operate upon computational objects representing infinite sequences. For example we need to be able to construct a sequence generated by doubling each element of another sequence. ToonTalk provides ways of expressing both the communication of data between processes and the coordination of the processes that were designed to be accessible by young children. Communication is accomplished by birds carrying objects from one place to another where it has its nest. An example is the Add 1 robot that repeatedly adds one to the first hole and gives a copy of the result to the bird in the second hole thereby generating the sequence of natural numbers on the bird's nest (Figure 3).

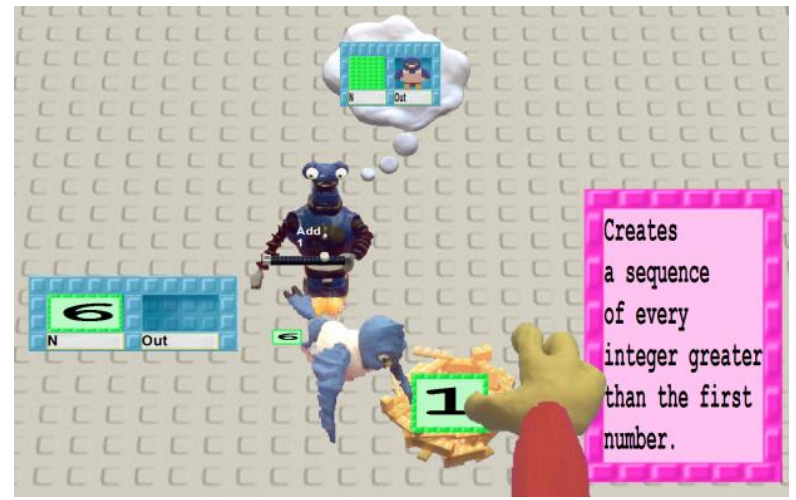

Figure 3 - The Add 1 robot producing the natural numbers on the nest (the first hole initially contained 1)

Robots will wait for deliveries to empty nests, thereby providing a way to express how processes should synchronise and coordinate their activities.

The concurrency and communication mechanisms afford learners with the means to express processes concretely as separate teams of robots repeatedly giving numbers to birds in a simultaneous mode. Each infinite sequence is made tangible as a nest where birds are continually delivering numbers. One can literally hold in one's hand a nest that over time has arbitrarily many natural numbers on it, and above all, one can perform operations upon these sequences of numbers.

To enhance the students' sense of infinity, we encouraged them to imagine what would happen if their robots continued to run forever. The finite limitations of real-world computational systems proved not to be a serious obstacle for the students to talk about, think about, and reflect on infinity because of the following features of ToonTalk: 
- It allows working with extremely large integers (the maximum value for integers is in the order of tens of millions of digits1) and slows down the execution as the numbers involved get extremely large.

- It can save the entire state of an ongoing computation, thus allowing the computation to be resumed on a different system, if needed (so the computation might go on forever even if the computer it is running on, does not).

- It can work with exact rational numbers, having a numerator and denominator that can have millions of digits, so that rounding errors or approximations can be avoided. This representation of rational numbers can help students explore with a deeper insight Cantor's surprising theorem about the one-to-one correspondence between the natural numbers and the rational numbers (see e.g. Dauben 1990).

Taken together, these features made ToonTalk an expressive environment for activities directed at exploring infinity with young students in a constructionist style.

\section{The activity design and the setting}

The activities were designed to lead students along a path that happens to resemble the historical development of the mathematics of the cardinality of infinite sets, from Galileo to Cantor and Hilbert. As is claimed by Piaget and Garcia (1989), traces of the cognitive processes found in the historical development of science may be similarly present in the development of individuals; in particular, when a moment of crisis (a scientific revolution - such as the one brought about by Cantor's set theory and the development of the mathematics of infinity) occurs, there is a break with the existing ideology and a new epistemological framework emerges. In this case, we chose to follow a similar path to the historical evolution and, as will become evident, it is often convenient in the analysis to draw parallels between the actual evolution of students' ideas, and the historical evolution of mathematical analysis.

Our Exploring infinity experiment was carried out within the WebLabs project (http://www.lkl.ac.uk/kscope/WebLabs/), in which students from six European countries explored topics in mathematics and science by building computational models and programs. The students published "web reports", typically containing their programs as materialised proofs of their reasoning (Mor \& Sendova, 2003). They read and added comments to each other's reports. The cardinality activities were field tested in UK and Bulgaria, and it is this data that we report on here.

In 2004, we tested an initial version of the activity sequence with two girls and two boys (10 and 11 years old) in a local primary school in UK. There were 26 sessions where about half the time was focused on exploring infinity. Based upon this testing we made a large number of relatively small revisions to the activity worksheets significantly improving the clarity of the instructions and questions. In 2005, we tested the second version in another school in England with a group of two girls and three boys (12 and 13 years old). There were 18 sessions of between 60 and 90 minutes on the cardinality activity sequence. This second version of the activity series was translated and tested, in that same year (2005), with five groups in Bulgaria, in two cities (Plovdiv and Sofia) with a total of 19 boys and 3 girls ( 9 to 13 years old). They met for 90 -minute sessions once a week for seven weeks in out-of-school context.

Despite the small scale of the field testing, we wanted to investigate the suitability of our learning trajectory for students from different cultures, ages, mathematical backgrounds, and school contexts. For example, the first UK study was with younger children with average mathematical abilities in a school context where a great deal of time was available for the children's participation. The second UK study was with older children, with above average mathematical abilities, in an after-school context with less available time.

The students from the first Sofia group were children of mathematicians and informaticians (colleagues of the WebLabs researchers, who had expressed interest and a wish that their children attend the WebLabs classes organised in the Faculty of Mathematics and Informatics at Sofia University). The second Sofia group consisted of children attending extracurricular mathematics classes in the National Mathematics and Science High School (a school for high-achieving

\footnotetext{
1 This contrasts sharply with the typical maximum integer size of $2^{31}$ (ten decimal digits),
} which are those that fit in 32 bits (with one bit used for specifying the sign). 
students in mathematics, admitted there after serious entry exams). Their age at the time of the cardinality activity sequence was $12-13$ years.

The Plovdiv groups engaged children from grades 3, 5, and 6, (9-, 11-, and 12- year old) who had the highest achievements in mathematics contests at regional and national level and had strong interests in working with computers. Only one student participated for a first time in computerbased activities.

All students followed an eight-step activity sequence. Each activity focused on a particular idea or challenge and consisted of two to five tasks which will be illustrated below. Some of the tasks were to train robots, others were to experiment with previously trained robots (often asked to predict the outcome before trying it), while others were to reflect upon previous activities. The reflective tasks were presented as printed worksheets with open-ended questions for the students to answer. Every activity included some reflective tasks which typically took less than a third of the session time. Interviews with a researcher (individually or as a group) were conducted aiming to encourage the students to make conjectures, propose their own definitions, and discuss their current understanding and intuitions. At three points, in particular, the students were encouraged to discuss their recent activities, write a group report, and post it on the project's web site.

The findings we report herein are based on six kinds of data gathered: (1) notes taken by researchers as the students worked (often in pairs discussing as they worked), (2) copies of the worksheets the students completed during the sessions, (3) the web reports published on the WebLabs web site by groups of students, (4) comments students posted on the web reports of others, (5) interviews with the students, and (6) computer logs of their actions. In the UK studies, notes were taken by one researcher observing typically four students. Detailed interpretation of this data was conducted by the authors. We are not making any claims of statistically valid general findings, nor any deep cognitive analyses of a small number of subjects. Instead we present nontrivial misconceptualisations and insights that we observed as the students did each activity.

\section{Exploring infinite sets: some results from the activity sequence}

\section{Computing the Even Numbers in Different Ways}

The first activity, Computing the Even Numbers in Two Ways, was designed to

- introduce infinite sequences as processes producing an infinite stream of numbers,

- introduce mathematical operations that treat infinite sequences as objects to be operated upon (e.g. doubling each term of a sequence, splitting an infinite sequence into two infinite sub-sequences, etc.), and

- $\quad$ encourage the students to reflect upon the seemingly paradoxical nature of infinity.

The students faced a paradox similar to the one Galileo described: how the sequence of the even positive integers can be placed in a one-to-one correspondence with the sequence of natural numbers and yet be a proper subset of it. (As we now know, this paradox became later the basis of a definition of an infinite set).

The activity began with the question: "Are there more natural numbers than even ones?" Coming from a specialised mathematics school, the Bulgarian students already "knew" the answer: that the even numbers are as many as the natural ones because "both are infinite" and they had been taught so by means of the traditional explanation on the board. They seemed comfortable with the even numbers being "twice bigger" and "equally long" with respect to the natural numbers (provided that every such notion would be strictly defined).

The students in both Bulgaria and the UK (who were from a standard 'comprehensive' school) then constructed programs to produce the even numbers in two very different ways. First they generated the natural numbers by training an Add 1 robot (Figure 3) that sends out successive integers accumulating on the bird's nest. They then used the output of Add 1 (the nest) as an input to a Doubler robot (which doubles every input element) thus generating the even numbers (Figure 4). 


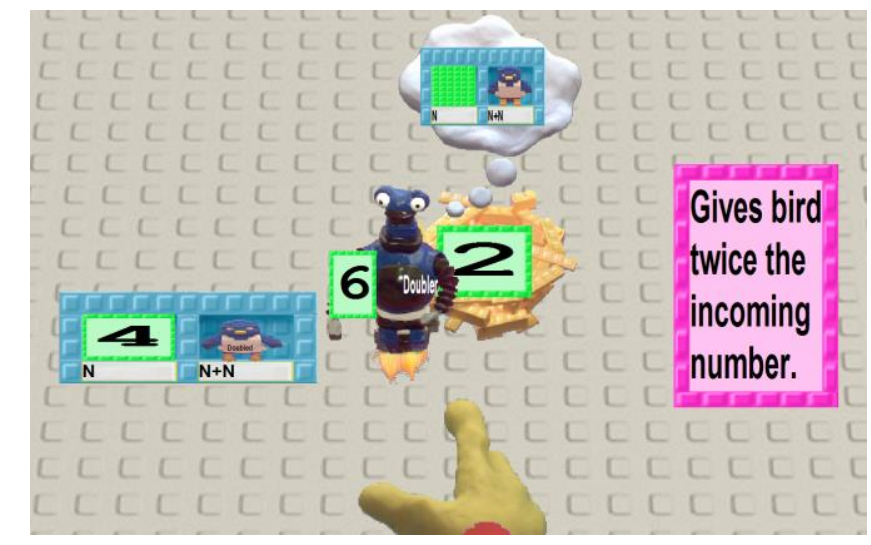

Figure 4 - The Doubler robot after doubling the first few natural numbers (the output of the Add 1 robot is accumulating on the nest in the hole in the box labelled $\mathrm{N}$ )

The second method chained the Add 1 to a Split robot which also receives the natural numbers but splits them in an alternating fashion into two nests: a sequence of the odd numbers (in nest A) and a sequence of the even numbers (in nest B) (Figure 5). We were surprised to find that only one child across all the sites seemed confused about what the Doubler and Split robots were producing as they consumed streams of successive natural numbers. The others demonstrated their understanding of their programs by answering questions about the process and its product or output. They made conjectures as to how these two programs can start with the same input sequence and produce the same output sequence despite the fact that one program discards half the input elements while the other uses every input element.

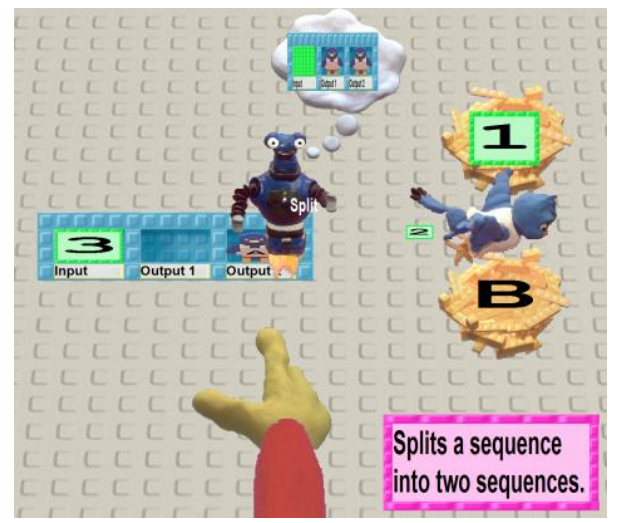

Figure 5 - The Split robot splitting up the natural numbers into two "piles"

One girl (Yana) in Sofia noted that in order to get the odd numbers she could edit the Doubler robot to also subtract by 1 . This Double then subtract 1 robot would produce the same numbers as those on nest A from the Split robot.

Next, the students were presented with the metaphor of dancing couples as a way to think about one-to-one correspondence. For example, the even numbers matched with the natural numbers produce a sequence of [2 dances with 1], [4 dances with 2], [6 dances with 3], and so on. In this activity, the students were asked to apply this metaphor to the infinite case of the output of the Doubler process:

Imagine that you are running the Doubler robot and that after the Add 1 robot gives a bird a number, it is copied and begins dancing with the output number of the Doubler robot. So 1 dances with 2, 2 with 4, 3 with 6, and so on. If this continued forever would every even number be dancing with a natural number?

Some of the students correctly believed that every even number would be dancing with a natural number. Others shifted in their responses first thinking it was so, then retracting. In answer to the question: "What does this tell you about how many even numbers there are?" again, there were differences in the responses: while some students wrote that the even numbers are infinite because "there is still another number up", others focused on them being "double the amount of natural 
numbers" (i.e. each number being twice a natural number), even if they readily accepted that every natural number would be dancing with an even number.

One of the main difficulties was that, as one of the English boys stated "some even numbers are natural", meaning most probably that "some natural numbers are even". Actually, the fact that the even numbers are a subset of the natural numbers was a problem for the dancing couples metaphor; in the words of an English girl, "because 1 is with 2, 2 with 4, 3 with 6, and so on". She said this, despite the fact that in the ToonTalk construction the bird copies the natural numbers. While some students accepted the idea that " 1 dances with 2 " and " 2 dances with 4 ", others became confused about who 2 is dancing with, thinking that 2 was paired with both 1 and 4 instead of realising that one instance of 2 is paired with 1 and another instance of 2 is paired with 4.

We did not anticipate this subtle confusion, since a number in ToonTalk is a virtual object located in space and hence cannot be in two places - it must be copied. Furthermore, the numbers of the first sequence could be thought of as labels (badges) of the boys (staying always in the left hole of the box), and the even numbers as labels of the girls (staying always in the right hole of the box). Still, we are now aware that it would have been even more convincing to put numbers on different colour pads. If for instance, the red natural numbers were dancing with the blue even numbers, then the red 1 would dance with the blue 2, and the red 2 - with the blue 4, and there would be no confusion whether " 2 " is dancing with both 1 and 4 . However, some pedagogical intervention might still be needed in that case to indicate that the colours were just a way of distinguishing one set of numbers from its copy.

In this first activity, we found all but two of the thirty one students quickly became proficient at creating and controlling infinite processes and inspecting the resulting output. The proficient students constructed explicit computational processes that operationalised the dancing metaphor and made the copying of numbers more explicit. The Bulgarian students and older UK group described and reasoned appropriately about these processes and objects at different levels. Sometimes they would describe the numbers on a nest as simply "even numbers". Other times they used high-level procedural descriptions as taking the natural numbers and doubling them, while occasionally they described the Doubler robot as multiplying by 2 . It was only the younger English students that often demonstrated preferred procedural descriptions based on the ToonTalk setting in which they were working: i.e. they would describe Doubler as a robot that copies a number, drops it on the original and gives the result to a bird (see Noss \& Hoyles, 1996 for the implications of this kind of thinking that relies on the setting where the activity takes place).

\section{Computing the Integers}

The second activity, Combining Infinite Sequences, was designed to

- $\quad$ provide a new mathematical operation on sequences (multiplying by -1 );

- introduce an operation that takes two infinite sequences and produces a super-sequence, and

- $\quad$ provide an example where the one-to-one correspondence between two infinite sets does not preserve ordering.

In this activity, students explored how the natural numbers can be placed in a one-to-one correspondence with the integers. They were faced with what at first glance looked like an impossible task: to make a sequence of all the integers. Most students initially attempted to program an ascending sequence and gave up. But all eventually did succeed in constructing robots producing the following sequence: $1,-1,2,-2,3,-3, \ldots$ To do this they first duplicated the output of the generator of the natural numbers; then they produced the negative integers by multiplying the elements of the second copy by -1 (using a Negator robot); finally they used the negative and the positive integers as input to a Merge robot (Figure 6). The students correctly described the result as the sequence of integers except for zero. (Later, zero was added in various ways to complete the set of integers.) Here again the students constructed programs that make concrete some counter-intuitive properties of infinite sequences, e.g. that a sequence with neither a smallest nor a largest element is constructible. The questions on the worksheets were intended, as in all the activities, to invoke reflection. 


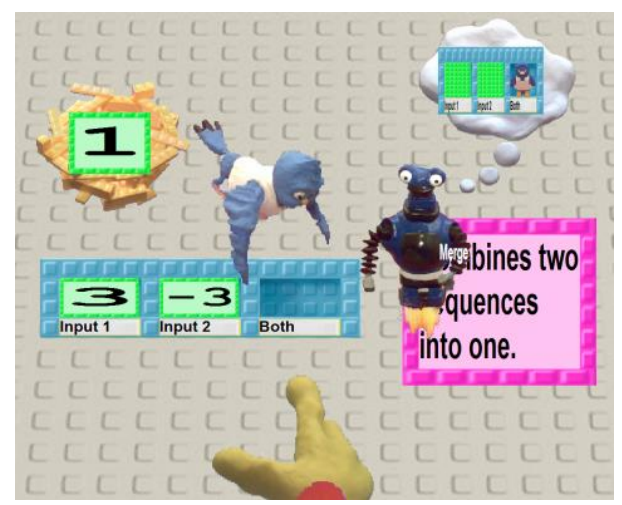

Figure 6 - Merging the positive and negative integers streams into one stream; on top of the nest is a stack consisting of $-2,2,-1$, and 1 (visible)

From later discussions and writings it is clear that many of the students thought there was a "natural" sequence of integers that in the middle looks like $\ldots,-3,-2,-1,0,1, \ldots$, and which can't be computed or described mathematically. Instead, they computed the sequence $0,1,-1,2,-2,3$, $-3, \ldots$ and answered questions about it but said the first sequence was more natural. As we shall see this concept of a natural ordering sometimes interfered with thinking about a one-to-one correspondence.

When faced with the question: "Can you see a way to get all the natural numbers to dance with all the integers?" a girl from the second English group said that if the natural numbers paired up with the positive integers then all the negative integers would be left over. After a discussion she wrote:

The numbers go on forever but for every natural number $(1,2,3$,) there are two integers $(1,-1,-2,2)$ so although you can write them out and they all pair up, every natural number is also one of the integers but there are twice as many integers.

Later that day she told the researcher, "It doesn't make sense but it must be right" since running forever exhausts both lists.

In a web report, a Bulgarian boy (Ivan) provided - together with a package of his robots and boxes (Figure 7) - an explanation of how his method of training the Merge robot made the integers "dance" with the natural numbers, thus showing that there are as many numbers in each set:

In my first version of MERGE1 the birds carrying the natural numbers and the [negative] integers were not synchronised so I decided to retrain it. In the last version, MERGE1 was connecting each dancing pair of integers and natural numbers in a 2-hole box and giving this pair to the output bird. So now it is clear that the integers are as many as the natural numbers!!!

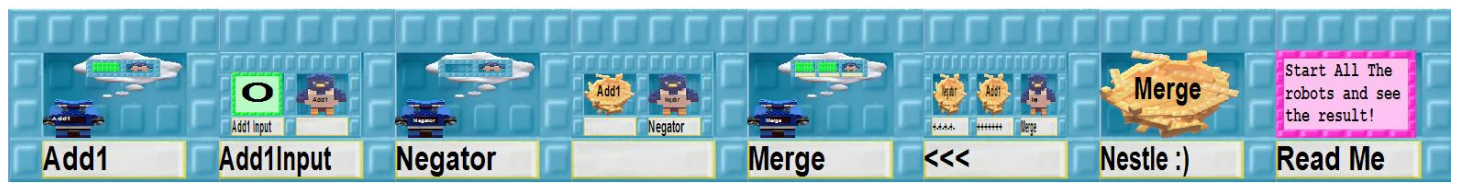

Figure 7 - Package (from a student's web report) of robots generating the integers

In this way, a robot can be seen as a constructive "proof" that there are as many natural numbers as integers!

Two students anticipated the next activity - having robots explicitly produce the one-to-one correspondence as a stream of data structures describing the pairings (an 11-year old boy from UK wrote: you could try and give a natural number to a bird and the other to the bird and make the robot make more birds to give numbers to).

\section{Comparing the "Size" of Infinite Sequences}

The third activity, Generating Different Infinite Sequences, was designed to

- $\quad$ enable the students to see that all the infinite sequences they devised could be paired oneto-one with the natural numbers,

- $\quad$ provide a metaphor for thinking about one-to-one correspondences, and 
- $\quad$ give the opportunity to construct and experiment with a computational operationalisation of one-to-one mappings.

In this activity, the students were asked to produce three different robots of their choice to produce different infinite sequences and discover whether their sequences can be paired one-to-one with the natural numbers. They had to operationalise the concept of a one-to-one mapping by constructing a Match Maker robot - based on the dancing metaphor presented in Activity 1. The students in the UK interpreted the Match Maker task as building a program that takes one infinite sequence as input and produces an infinite sequence of pairs of the elements of the sequence and the sequence of natural numbers. The students in Bulgaria instead made Match Maker robots that took two infinite sequences as input and produced a one-to-one correspondence between the respective sequences. Note that given two infinite sequences $A$ and $B$, the UK students were able to use their Match Maker robot twice: once to make a pairing between $A$ and the natural numbers, and then between the natural numbers and $B$. These pairings were then joined to produce a direct pairing between $A$ and $B$.

The students posted their robots and constructions on the WebLabs project's web site. Then they downloaded the robots and sequences made by other students (either in the same or other country), ran them, and had to describe them and answer questions.

At the end of the activity, each of the participating groups had to post a web report on the WebLabs system. For example, the younger English students gave low-level ToonTalk-situated descriptions of how they constructed their number sequences, and of the Match Maker robot:

This robot [Match Maker] it gives the number to the bird and the bird adds it to the 1 or the other number and makes it into a 2. It copies the box and takes the box off to its nest and then it pulls out a number 10 and then starts counting by 10.

In the web report, the students shared their discussions and consensus of their ideas, in answer to questions such as the following:

- When can you say two infinite sequences are the same size?

- Can you give an example of two infinite sequences, which according to you have the same size?

- Can you give an example of two infinite sequences of different sizes? If not, why not?

- Are there infinite sequences that have so many terms they can't be counted?

When Cantor translated fundamental sequences into the language of sets, questions such as these (using the word "set", instead of "sequence" as above) were the core of Cantor's work on infinite sets that led to the development of the formal concept of actual infinity, and the subject of a lively mathematical debate in the $19^{\text {th }}$ century (Dauben, 1990).

In the activity sequence, we chose to focus upon infinite sequences rather than the mathematically more conventional infinite sets for many reasons. Sequences are relatively easy to compute while sets are much more problematic computationally. The very existence of an infinite sequence implies that its elements are "as many" as the natural numbers. But to generalise the relationships between sequences to relationships between sets the sequences cannot contain any duplicate elements. We designed the activities and computational resources to address this problem.

In this activity the students successfully built an infinite process for describing a correspondence between the elements of two sequences. Some of them saw the need to be sure that the sequence had no duplicates for the results to be a valid one-to-one mapping. The Bulgarian and the older English students were able to reason successfully about their programs running forever, while the younger English students seemed confused. All but these younger English students correctly answered questions about the application of their mapping program to sequences they and their fellow students generated. In some of the later activities where the students applied the same program to similar tasks they failed to show the understanding they showed here. With hindsight we believe that more time should have been devoted to reviewing past work before reapplying it to new situations.

As illustrated below, both the UK and the Bulgarian students demonstrated surprisingly sophisticated reasoning on the first question involving a newly coined notion "sequences of the same size". In their group reports, the second year English students came up with two different definitions of the concept of size applied to infinite sequences and then explained how by the first definition all infinite sequences are the same size, while the second definition imposes strict conditions: 
It depends what is meant by 'the size'. It could be that the size is the total length of the sequence or the distance between terms. For the total length of the sequence, they're all the same size, as they all go on for infinity, but if "size" refers to the distance between the terms then if they start on the same number and each term is the same distance apart in both sequences, they will be the same size.

Thus, in response to the question asking for examples of two infinite sequences of different size, they provide different answers depending upon which of their two definitions is applied:

If the total length of the sequence is what 'size' means then no because, as stated above, all sequences go on forever. If, however, size means 'the distance between consecutive terms' then it is very easy. Of the sequences $2 n$ and $3 n$, where $n$ is every natural number, $2 n$ produces more numbers before 100 (for example) than $3 n$ because the terms aren't so far apart.

One of the groups in Sofia followed a very similar reasoning. At first they could not give examples of same-sized infinite sequences "because they are infinite and we don't know the number of their terms". This led to an interesting discussion amongst the students of what was meant by "size", proposing multiple mathematical definitions - as in the case of the English group - and reasoning about them:

Teddy: ["Size"] could be the difference of the consecutive terms of the sequence.

Mitty: We could define three things for determining when one sequence has a greater size than another: the number of the terms; the value of the terms; the distance between consecutive terms

Teddy: I agree, if we could say that there are more terms in a sequence than in another one, the first sequence would be of a greater size.

Mitty: On the other hand, if the distance between the consecutive terms of a sequence is small, i.e. if it grows slowly, and the [corresponding] distance between the consecutive terms of another sequence is bigger we could say that the second one is bigger.

Teddy: This would mean that there are infinities of different size. I think that the number of the terms in a sequence could be bigger than the number of the terms in another one though...

Thus in answer to the questions asking for examples of infinite sequences of same/different size, the students came up with the following discussion:

Ivan: Well, take these two sequences:

(1) $1,2,3,4,5, \ldots$

(2) $30000,50000,70000, \ldots$

If we mean 'the distance between consecutive terms', then the sequence (2) is of bigger size than (1). But if we think of the number of the terms we couldn't say that one of them is "bigger".

Mitty: Here are my two sequences:

(3) $1,2,3,4, \ldots$;

(4) $2,4,6, .$.

I would say that these sequences are of different size but of equal length since the terms of (4) are bigger than the corresponding terms of (3). But they are equally long since neither of them ends - after each pair of odd-even numbers there follows another pair of odd-even numbers and after each even number there is another even number - both sets are infinite.

Teddy: Yes, but in addition, the distance of the consecutive terms of the first sequence (3) is smaller than the corresponding distance of the second one (4) and we could say that the second one grows faster and is bigger than the first one.

These students were capable of inventing and correctly applying multiple definitions to match different intuitions about how to compare the size of infinite sequences. Mitty does something very interesting: rather than give multiple definitions of the concept of size, he calls one concept "size", and the other "length".

As we see in the above transcripts, in order to define the size of an infinite sequence (set), both the English and the Bulgarian students discussed the number of terms in the sequence (or "length" of the sequence) which as explained by the Bulgarian students could not give enough information "because they are infinite and we don't know the number of their terms"; or in the English terminology "go on forever". They both interestingly and alternatively also considered the idea of distance or difference between consecutive terms (which can also relate to the rate of "growth" of the sequence, as expressed by Mitty, in terms of how quickly the values of the terms increase - i.e. 
the rate of divergence). In order to distinguish between infinite sequences, and find ways in which they could be of different size, they came up with interesting possible definitions, and even refined them. For instance, Ivan started with saying:

A sequence grows faster than another one when the difference between two consecutive terms of the first one is bigger than the difference of the corresponding terms of the second.

After consecutive refinements provoked by the researchers he reached the following algorithmic definition:

We should look at the sequence of the differences of the differences. If in both cases these differences are growing we could say nothing. We should look at the differences of the differences of the differences so many times until one of them stops growing or becomes decreasing

In fact here Ivan is talking about finite differences of a higher order in his own words!

Later, in the Bulgarian group, in answer to the teacher's question: "Can we build a robot (robots) which taking the natural numbers as input would produce two sequences with different number of terms?" Ivan replied:

Ivan: Yes, we could make the Counter [robot] give 1, 2, 3, ... to Robotl to produce $2,4,6, \ldots$ and to Robot 2 to produce a couple of numbers for each natural number, e.g. for input 1 it will produce 1, 2; for input $2 \rightarrow 2,3$; for input $3 \rightarrow 3$, 4; etc. Thus the second sequence 1, 2, 2, 3, 3, 4,... will have twice as many terms.

This student seems to have invented yet another way to compare infinite sequences by comparing the number of elements generated after the same number of iterations (though this doesn't generalise to sets).

Not all the students debated the concept of size. The oldest Bulgarian group in Plovdiv, gave as an example of two sequences of the same size: $\{1,2,3,4,5,6, \ldots\}$ and $\{2,4,6,8, .$.$\} , explaining that$ "they are formed in a similar way" and "for each number of the first one we'll be able to find another one from the second sequence". But they did not address the question whether every number from the second sequence maps to a number in the first.

In contrast, the group of younger students in England seemed to think that two sequences are of different size if they have different elements, without referring to the concept of a different number of elements. They wrote they could give examples of infinite sequences of different size by changing the values of the terms in the set:

... because you can just times by different numbers. This makes the answer to any other sequence different. The sequence starts with different numbers.

And they wrote as examples of their procedure the following:

$(1 \times 2, x 2, x 2, x 2$, etc $)$

$(+45 \times 3)$

Note their invention of a notation for describing sequences: The first sequence starts by multiplying 1 (the initial number) by 2 and then repeatedly multiplying the previous element by 2 to obtain the next element. The second example describes a sequence where each element is obtained by adding 45 to the previous element and multiplying the result by 3 . They neglected to give an initial value here.

One of the questions on the Activity 3 page was: "Does the sequence contain any duplicates?" The two girls from the older English group discussed this for several minutes, and observed that in a sequence you add 1 (then do things to the number), so you start with different numbers and that would mean you always get different numbers; therefore there are no duplicates. For example, for one of their sequences which was generated by squaring the natural numbers then subtracting 20 from each element, they wrote:

No [it doesn't contain duplicates] because every time we start with a different number $(1,2,3)$ the square -20, you still have different numbers.

One of the girls later explained that if there were duplicates in a sequence, then the one-to-one mapping wouldn't be correct. This technicality led this girl to invent sophisticated arguments about why her sequences were free of duplicates.

Below we give these girls' written answers to the questions that followed; the response to the first question is consistent with their previous answer: 
If all the robots run forever, will each number in the sequence be dancing with a different natural number? Explain.

Yes. As long as Match Maker adds 1 to the matching number every time no number will be repeated and every number will be included.

Will all the natural numbers have a different dancing partner? Explain.

Yes. But only if the original sequence has no repeated numbers, then some partners would be with 2 natural numbers.

This was the only group whose response indicated an understanding of why duplicates in a sequence interfere with constructing a one-to-one correspondence between the elements of a sequence and the sequence of natural numbers.

In answer to the question: "Are there infinite sequences that have so many terms they can't be counted?" some students in the first-year English group thought there were uncountable sequences until one of them said there couldn't be since you can train robots to count numbers in any sequence.

This answer demonstrates some insight into the nature of infinite sequences and their relationship with infinite computational processes. Note that a corollary of Cantor's theorem about the uncountability of the set of real numbers is that it is impossible to construct a sequence of all the real numbers. So these students 'rediscovered' in fact the term "countable" for any set whose elements can be put in 1-1 correspondence with the natural numbers.

\section{Generating the Rational Numbers}

The next three activities in the learning trajectory we designed were intended to lead the students in exploring the set of rational numbers and its correspondence with the set of natural numbers. The one-to-one correspondence between the rational numbers and the natural numbers is particularly surprising since the rational numbers are everywhere dense (i.e., between any two rational numbers there is always another one, and therefore - infinitely many rational numbers), while the natural numbers are discrete.

Let us make a small diversion and see how Serge Lang (considered by many of his Yale students to be one of the greatest teachers of mathematics) discussed this fact with 11th graders from Toronto in his talk on infinities (Lang, 1985). While they had grasped quickly the one-to-one correspondence between the natural and odd/even numbers, then - between natural and integers, nobody believed at first that this was also possible for the rational and natural numbers. A student's first "counter argument" was that the fractions between two integers are infinite...Another student added: You have an infinite number of denominators and an infinitely many numerators. So how can you line it up when you don't know how high this is going to go? To which Serge Lang answered: Would my failure to line them up be due to my stupidity... or because of the mathematics? So I am simply questioning you: How do you know if it is built in the structure of mathematics, or if it's built into the accidental thing that I am not clever enough to do it? ... All you have said is that the method which I used for the even and odd numbers doesn't apply to the rational numbers. How do you know that if I am clever enough, I can't find another method?

After that Lang constructed with the help of the students an array in which each row has sequences of fractions with the same denominator, and the denominators increase vertically. A discussion followed and the students, with the support of Lang, figured out how to enumerate the fractions! But as he said in conclusion: It's tricky!!!

In our case we wanted for the kids to make this counterintuitive correspondence tangible. Here is how we proceeded.

\section{Generating all fractions in $(0,1)$}

This activity was designed to

- begin the exploration of the cardinality of the rational numbers, and

- provide the surprising experience of making a one-to-one correspondence between the natural numbers and the proper fractions. 
It began with the construction of a robot to produce a sequence of all the rational numbers between 0 and 1. The students constructed a Next Numerator robot that outputs a proper fraction and then increments the numerator by one. This robot repeats until the numerator and denominator are equal. Then a Next Denominator robot resets the numerator to 1 and increments the denominator by one. These robots, when combined, produce the sequence $1 / 2,1 / 3,2 / 3,1 / 4,2 / 4,3 / 4,1 / 5, \ldots$ Removal of duplicates (e.g. $2 / 4,3 / 6,4 / 6$ ) is handled by robots that we provided, though the challenge of constructing a program to remove duplicates is not beyond the capabilities of the advanced students.

All the students were able to construct the program for generating all the proper fractions, to describe the underlying algorithm, and correctly characterize the output. They were able to argue why it would produce all the fractions between 0 and 1 if it ran forever, that the sequence produced includes all the proper fractions with denominator 2 , with denominator 3 , and so on. Several of them wondered about 0 and 1 and saw how they could start their process so that it began with 0 . They seemed certain that 1 would never be included since the process is always generating fractions with numerators less than the denominators. The question of whether 1 was included became important when later they combined the proper fractions with their reciprocals and had to decide if they had obtained all positive rational numbers.

On the worksheet students were asked "Do you think all the fractions between 0 and 1 are countable?" and requested to write down how they would convince a younger child of their answer.

Unlike the similar question about even numbers and integers, most of the students answered in the affirmative. At first they didn't think it was surprising that the proper fractions between 0 and 1 were countable, until they realised that includes the reciprocals of the natural numbers plus, as they said, "lots more".

Some of the answers from the younger English students were:

\section{- Yes. I would say that ToonTalk can count all fractions, every one, even though there is lots. \\ - Yes. If we got all the fractions, we could spend forever counting them but it would be possible.}

Note that these students referred to ToonTalk counting the elements of the infinite sequence, which indicates how situated their ideas were with reference to their experiences during the activities. They had no difficulty applying their program to operationalise the one-to-one correspondence with the natural numbers, so they saw quickly that the output of their robots was countable since you can take the first fraction off the nest and say "one", then the next and say "two", and so on. (The fact that the set of natural numbers and the set of proper fractions are disjoint means that there is no chance of confusion about the same number appearing in two mappings.) So they saw that a process that ran forever could count all the proper fractions (even though the rational numbers are everywhere dense).

The way in which the fractions were constructed, the ordering of the sequence seemed sensible (the halves, the thirds, the fourths, etc.), even if it isn't in ascending order, and the issue of density is perhaps not obvious.

At the same time, the fraction presentation (decimals v. quotients of a numerator and denominator) seemed to alter the way the students thought about this activity. During their discussions, a boy and a girl from the English group, talked about all the numbers with one decimal place, then all with two decimal places, and three decimal places, and so on. This is a variant of their process that also generates the proper fractions. The girl said of the sequence expressed as decimals: "It doesn't appear to make sense but it must, since we made it". Thus, the students' active engagement in the construction process helped them give meaning to the relationship, despite the misleading representation (which didn't "make sense" - as ordinary fractions would).

However, due at least partly to the decimal representations used, the English students found it difficult to extend their interpretation of the countability of the rational numbers between 0 and 1 to all the positive rational numbers. They wanted a more orderly sequence. This led to an insightful discussion about the difficulty of producing the first element, i.e. the smallest fraction greater than 0. (In a later session, at the suggestion of the researcher, these students rebuilt their sequence so that ToonTalk would display the output numbers as ordinary fractions rather than as decimals, and they expressed they liked it better.) 
Were we to do further studies we could alleviate the students' difficulties with decimal representations by introducing a new activity where the students produced the tenths $(1 / 10,2 / 10$, $3 / 10, \ldots 9 / 10)$, and then the hundredths $(1 / 100,2 / 100,9 / 100,10 / 100,11 / 1000, \ldots 99 / 100)$ and so on. The duplicates could be removed from this sequence by testing whether the numerator is divisible by 10 . We would encourage the students to ponder why this produces all the rational numbers between 0 and 1 while they earlier had produced the same set of numbers by generating all the fractions of successive numerators.

\section{Generating all rational numbers in $(1,+\infty)$ and mapping them to those in $(0,1)$}

The next activity, Generating a sequence of all rational numbers greater than 1, was designed

- to challenge the students by guiding them to construct the rational numbers greater than 1 , while simultaneously operationalising a one-to-one mapping with the rational numbers less than 1 , and

- to encourage the students to reflect on the relationship among these two sets.

In this activity the students had to produce the sequence of reciprocals of the proper fractions, i.e. the rational numbers greater than 1 . To induce reflection and confrontation on the intuition we included in the activity two questions that describe commonly held misconceptions:

Pat says: There must be more rational numbers between 1 and infinity than
between 0 and 1 . Is Pat right? Explain.
Sally ... says: "There must be the same number of rational numbers from 0 to
1 as there are from 1 to 2 . And from 2 to 3 . And 3 to 4 . So there are an infinite
number of intervals of the same size as 0 to 1 . So there must be an infinite
times more rational numbers greater than 1 , than between 0 and 1. " Do you
agree or disagree with Sally? Why?

In response to the opening question the first cohort of English students wrote:

No because there is an infinite amount of numbers between 0 and 1. But also between 1 and infinity.

This correct answer is particularly interesting since the students contradict this later on in this activity. They seemed to believe - unsurprisingly! - that all infinities are equal, the position commonly held before Cantor. "Sally's argument" was very confusing for most students. In the second English group, one girl wrote:

[I agree with Sally] sort of. But you can't have $\infty \mathrm{x} \infty$, so what is she going on about?

The researcher then asked whether the proper fractions could be made to dance with the rational numbers greater than 1 . The first idea this girl came up with, was to start with the lowest number between 0 and 1 and have it dance with the lowest number greater than 1 and so on. A fellow student asked her how she could get the smallest number like that. She saw this was problematic and then she suggested they start with those with one decimal place, then those with two decimal places, and so on. This seemed to be a fine way to get the numbers between 0 and 1 dancing with those between 1 and 2; in response to a question about all the numbers greater than 1 , she started a complex explanation on how she could do that too.

But then the fellow student pointed out they could use the sequences they had previously generated: he seemed to mean that $1 / 2$ would dance with $2,1 / 3$ with $3,2 / 3$ with $3 / 2$, and so on. It is curious that despite having just run a program that operationalises this mapping, these students were swayed by Sally's arguments and it was only after a long discussion that their recent programming experience led to an insight on the topic.

In other words, despite having computationally constructed a one-to-one mapping between the natural numbers and the positive rational numbers, and another such mapping between the rational numbers in $(0,1)$ and those in $(1,+\infty)$, their intuitions that the size of the set of positive rational numbers must be larger, confused them.

A boy in Bulgaria (Ivan) had planned, initially for this task, to modify the robots that generate all the proper fractions but when a teacher asked if there was an easier way, he changed his strategy, which he explained as follows: 
So, I'll use the team of robots generating the proper fractions to feed my new robot. I will divide 1 by each of the coming numbers and give the result to a new bird.

This episode is a nice example of where the practice - common in programming - of reusing old programs can be combined with a mathematical insight. Had Ivan trained new robots to generate "improper" fractions rather than generate them by computing the reciprocals of the output of the previously built proper fraction generator, the one-to-one correspondence between the proper and improper fractions would not have been available. In other words, had he followed his original plan, he would have missed the opportunity to program a one-to-one correspondence that both constructed the rational numbers greater than 1 and "proved" that the rational numbers between 0 and 1 have the same cardinality as the rational numbers greater than 1 .

When Ivan was presented with Sally's argument, although he thought initially that it sounded reasonable, he felt uneasy about the statement. His teacher tried to help with a picture on the blackboard (Figure 8) and said:

In which of the two segments are there more points? The logical answer would be "in the longer one". But let us connect their ends and make a triangle. Now try to find a point in the longer segment for which I would not be able to find a "partner" in the shorter one. See?

(a)

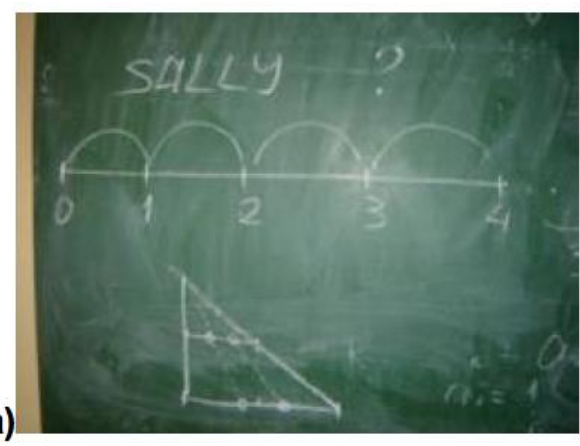

(b)

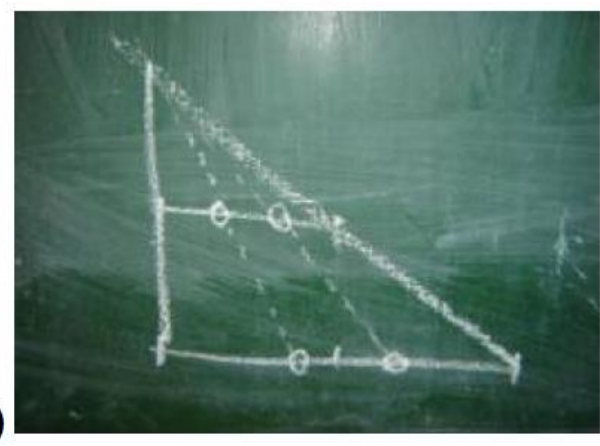

Figure 8 - Bulgarian teacher's representation of the one-to-one mapping between two segments: (a) Full picture on blackboard; (b) Close-up

To this Ivan replied:

I see your point. So you want me to find a fraction in the interval $(0,1)$ for each fraction greater than 1 that Sally would choose. In other words, we could combine each number of Sally (greater than 1) with a number less than 1. That does not seem very difficult, for example, if she chooses 205/3, I'll answer with 3/205, if she chooses 101/11, I'll take $11 / 101$, and so on.

In this way, when considering the problem in fractional notation, Ivan saw the one-to-one correspondence. He then implemented the idea using his Match Maker robot to explicitly produce the pairings (Figure 9). He also used a game-theoretic way of thinking about the one-to-one mapping: Rather than describing the mapping as an object, he presented a general strategy for producing a corresponding element to whatever "move" the opponent makes. Also, unlike the experience of the English students, clearly Ivan's approach was facilitated by the numbers' fractional representation that he used. 


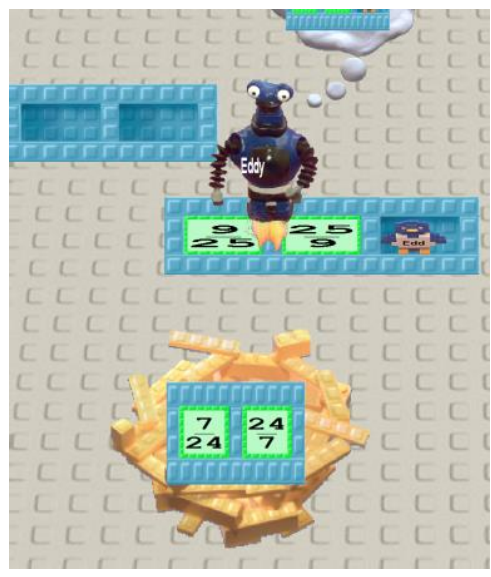

Figure 9 - Ivan's Match Maker robot pairing up fractions and their reciprocals

\section{The final steps to generating all the rational numbers}

In the next activity, Combining Infinite Sequences - Part 2, by combining previous sequences, students could produce a sequence of all the rational numbers. This activity was designed to

- repeat in a new context the lessons of combining the positive and negative elements, and

- give a sense of accomplishment in generating a sequence of all the rational numbers.

Using the Merge robot, the students began combining their sequence-generating processes to produce the rational numbers between 0 and 1 , and the reciprocals of those numbers. As a result they produced an interleaving of the two sequences that generates the positive rational numbers. And then, by combining the rational numbers with the negative rational numbers, they produced a sequence of all the rational numbers. Moreover, each step helped them discover that their Match Maker robot can still produce a one-to-one correspondence between the natural numbers and the intuitively "larger" sets of rational numbers.

Although none of the students had a problem seeing how to produce the positive rational numbers, many of them did realise that including 1 might be a problem:

The students in the first English group thought that the result of their merged processes would include 1 (only once); while the second group thought 1 would be missing from the result. Aside from this issue about 1 , they all saw that all the positive rational numbers would result. (A girl in the first group explained that if the robots ran forever, one would get infinity divided by infinity; when she was asked what infinity divided by infinity would be, she answered infinity.)

When Ivan, in Bulgaria, was asked if his robot constructed during a previous activity could produce 1, he responded:

Let us consider the robot merging the sequences of the rational numbers smaller than 1 and bigger than 1. We can't get 1 in the first sequence since the numerator is smaller than the denominator. As for the second sequence - it depends on the first; therefore, the numerator is greater than the denominator. In neither of the cases we would get equal numbers as inputs, therefore we would not get 1 .

When the students in the third group in Plovdiv (12-year olds), were asked to produce a single sequence which would "contain all the elements of the sequence of rational numbers between 0 and 1, and that of those greater than 1", a lengthy discussion followed between two students, and a debate as to when the sequence would stop and if it would include 1:

Theodor: This is simple - we had a robot that was merging two sequences.

Victor: Why not construct a new robot which would take a 3-cell box (with birds) and we could even count which number of term we have reached. ... If you count the consecutive terms you could compare the number of rational numbers between 0 and 1, and those greater than 1. [...] take a number from the first sequence and a number from the second sequence while there are numbers in both places. Right? If one of the birds stops bringing numbers then the robot will stop and we'll even know how many numbers there are in the sequence making it stop. Thus we will know both that we have counted the number of terms and like that we can compare these sequences.

Teacher: So, when do you expect your robot to stop...? 
Victor: I haven't thought of this. Maybe it would stop because of the first one, the one containing the proper fractions... The numbers greater than 1 are - oh! ho-ho-ho infinitely many! So it wouldn't stop because of it, right? ... Let's see. Maybe the last number would be 0.99 .

Theodor: Nonsense! This is not true! I can show you that there are more numbers after that on the number line, that are closer to 1. [He draws on the board the number line, leaving a big space between 0 and 1 . He then writes 0.99 at a distance $3 / 4$ of the interval $[0,1]$. Then he depicts the numbers $0.991,0.992$, etc. Then 0.9991 and so on, finishing with several numbers with 6 digits after the decimal point.] Whatever number you tell me [between 0 and 1], I could always find a number that will be bigger than yours and closer to 1 .

Victor: We already realized that the first robot will never reach 1. And the other one starts from a number greater than 1. So we are never going to receive 1 although we'll approach it closer and closer, sometimes just a hair apart but we'll never get exactly 1. [He points at the numbers with 6-7 digits after the decimal point, depicted previously on the board.]

Theodor: If we make the second sequence to start from 1, it will appear just once and no more...

In the above excerpt, we see Theodor clearly explaining the density of the rational numbers. The two boys also realise they could get arbitrarily close to 1 , but not reach it; in order to make sure 1 is in the sequence, Theodor then proposes a modification of the procedure generating the sequence of all positive rational numbers.

\section{More on the countability of rational numbers}

In the same conversation as above, the two students from Plovdiv also discussed the size and countability of the rational numbers. They began comparing the size of the sequences between 0 and 1 , and that of those greater than 1 (see the transcript above), concluding that they would both have the same size but would be infinite; then they considered all the rational numbers which they knew they could get by means of the Negator and Merge robots:

Theodor: Once both of them are infinite, they should be equal!

Teacher: What do you mean by that? Are you saying that the first sequence has as many terms as the second one?

Victor: I am not sure. I don't think this is a YES-NO question. Or at least we can't assess it now. If we run the robot it could work till tomorrow without stopping... So till then, there will be equal number of terms in both sequences. But after that? We would not be able to tell if the robot would stop somewhere and even if it does this would be somewhere in the infinity and our life would not be long enough to wait till then (if of course our computer does not crash before that...)

Theodor: That means that they are equal till infinity :), i.e. to a certain infinite member?

Teacher: Now, we could get all the rational numbers by means of the Negator and Merge robots. Do you think they are countable?

Victor: No, as we saw in the previous case, we can't count them up because you could always find a number after each term of the sequence. As far as we want...

Theodor: More so that we are having the negative numbers in addition... That means that they become twice as many... The positive rational are infinitely many, and if you combine them with as many negative, what would you get.. twice infinity! Who could tell me how much this is? [laughing]

Victor: Too much!

Teacher: How would you convince someone?

Theodor: To convince him? Why should I exhaust myself - I would show him my robots, he would see that that's exactly what we need and I would let him wait until he gets convinced that this is really the case :) I don't need to get tired and talk and argue with him... Thus when he gets tired from waiting, my job will be done!

As we see, for these students, despite the fact that they have come up with a means of sequentially generating the rational numbers (and therefore of indirectly putting them in a one-to-one correspondence with the natural numbers), the density of the rational numbers still presents an obstacle to the idea that the rational numbers can be counted. On the other hand, they do conclude that the rational numbers will be infinite, and in that sense not countable to a certain number (a 
different definition of countability than the formal one2), which is also what some English students debated:

When confronted with the worksheet question:

Can the rational numbers be counted?

If not, why not?

If so, how would you convince someone?

A boy and girl in the second English group wrote:

It depends upon what 'counted' is. You could start but never finish.

A discussion about the countability of the rational numbers followed, with four students in the English group. A girl argued that if you had two people you could count it, because one would count the negative numbers, while the other counted the positive numbers. When asked if they could be counted in some other order, she replied this was the natural way. However, on the computer screen, the first dozen computed terms were laid out $(2,3,1.5,4,1.333 \ldots, 5,2.5$, $1.666 \ldots, 1.25,6, \ldots)$; she said she could also count those, although she felt it was strange.

This was followed by a discussion of whether there were twice as many numbers between 0 and 2 than between 0 and 1 (similar to the ideas contained in "Sally's argument" in the previous activity). This led to a discussion of whether one can sensibly talk about $2 \mathrm{x} \infty$ or $\infty \mathrm{x} \infty$. The researcher then asked about the numbers between 0 and $1 / 2$, and a girl said there was an infinite amount; she went on to say there were an infinite number of numbers between any two rational numbers (the subject of the next activity).

The result of doing this activity once again highlighted a discomfort that students had with sequences producing numbers in a strange order that we observed in the two groups of English students. Despite having constructed a process to demonstrate a one-to-one mapping of the rational numbers and the natural numbers, confusion about how to handle both the negative and positive rational numbers arose. In the process of reflecting upon this activity the students revisited what it means to be countable, to run forever, and whether it makes sense to talk about multiplying infinity by other numbers, including infinity.

\section{On the density of rational numbers}

The next activity was designed to

- $\quad$ encourage the students to explore the density of the rational numbers, and

- provide an understanding of how the rational numbers between 0 and 1 can be manipulated arithmetically to produce a sequence of all the rational numbers between any two numbers.

The activity entailed training a robot to map the rational numbers between 0 and 1 to any interval between two rational numbers. The students took the rational numbers in $(0,1)$ and expanded, scaled, and shifted the interval to produce the rational numbers between any two rational numbers.

To the question of whether there always is a number between any two rational numbers, both the first and second year English groups answered affirmatively. The first year group wrote:

Yes. Because there is a half number between two rational numbers.

The second year English group referred to decimal places and then presented an example of averaging:

Yes, because you can always go on to the next decimal place. A number between 1.4 and 1.5 is 1.45 .

However, it is not clear how one can "go on to the next decimal place" if there were infinite repeating decimals.

As to the question: "How many numbers are there between any two rational numbers?" both English groups answered "infinite". The first year English group wrote:

$2 \quad$ After analysing the data we noticed the ambiguity of the word "countable" caused confusion. A revised version of the activity materials should be revised to avoid this. 
There are infinite numbers between two rational numbers because you can halve anything.

By "halve anything", they mean finding the number halfway between two numbers, i.e. the average. In contrast, the second year English group focused on the number of places in the decimal expansion to justify their answer:

Infinite, because there is an unlimited number of decimal places that you can use.

Here is how some students in Bulgaria also responded to this question:

Ivan: If [the rational numbers in] all the intervals can be generated from [the rational numbers in] the interval $(0,1)$ then there will be as many rational numbers as in the interval $(0,1)$. There is a one-to-one correspondence between [the rational numbers in] any interval and [the rational numbers in] in the interval $(0,1)$.

Teacher: If we have two arbitrarily chosen rational numbers do you think there is a rational number between them?

Teddy: We can always fit in several numbers between two rational ones...even infinitely many...

Note that Ivan uses a one-to-one correspondence to make a sophisticated argument that there are an infinite number of rational numbers between any two rational numbers. The English students also correctly answered this question but relied upon the process of averaging or the representation of decimal fractions.

Both groups of English students operationalised the concept of countability in terms of infinite computational processes. In answer to the question: "Are the rational numbers countable?" the first year English group said:

Yes. Because ToonTalk robots can count up to infinity.

While the second year English group wrote:

No. Well, kind of, but you could never finish counting them. One way of counting them would be to match each one with a natural number. Another way could be to go through each of them in your mind (or get a computer to do it) and recognise each as a number.

The students were then asked to reflect on whether the interval $(0,1 / 1000000)$ can be put in correspondence with any other interval. Then, in answer to the question: "Are all the rational numbers between 0 and 1/1000000 countable?", the first year English group again went into a debate of what "countable" means and showed a confusion between counting the elements of a sequence (or set) and counting the digits of a decimal expansion (perhaps they thought only "readable" numbers are countable and infinite decimals are not readable); however, in the end they again operationalised the concept of countability in terms of infinite computational processes

Countable means numbers you can read and understand. So if you [have] done 1/3 [it] would equal 0.333333333333 etc so that would not be countable.

The second year group used the idea of a one-to-one correspondence (pairing) with the natural numbers to answer the question of the countability of the numbers between 0 and 1/1000000:

Each could be paired with a natural number but you could not start at the lowest and finish at the highest after pairing each and every one. If you were counting in a different way you could count them in groups according to the denominator i.e. all the ones with 1 as a denominator, all the ones with 2 as a denominator, etc...

Note their preference for a one-to-one correspondence that is "natural", i.e. where the elements of the sequence are ascending. It is interesting that they give here a way to count the rational numbers between 0 and 1 , not between 0 and 1/1000000, but the basic idea can be adapted to this task.

The students were asked: Could all the rational numbers dance with all the rational numbers between 0 and $1 / 1000000$ ?

To this question, the first year English group wrote:

No. Because when we [have] done dancing with whole numbers, they were always dancing with numbers that are different from their selves.

Note that what they say about the earlier activities with integers isn't true since they were making the integers dance with the natural numbers. Apparently they find it confusing to allow the same number to appear in more than one mapping.

The second year English group couldn't reach a consensus: 
Allen: I think not, because if we define the number of rational numbers between 0 and $1 / 1000000$ as $x$, then the number of rational numbers between -infinity and +infinity will be more than $x$.

Susan: I think you could start to match up the positive rational numbers with the rational numbers between 0 and 1/1000000 but you would never get higher than 1/1000000 because there are infinity numbers between 0 and 1/1000000 so you wouldn't finish them. I have no idea how to include the negative rational numbers - where could you start counting?

Tom: I think you can because it's possible to make all the rational numbers dance with all the natural numbers. And it's also possible to make the rational number between 0 and 1/1000000 dance with the natural numbers: 'A' can dance with the natural numbers. For example, a number to dance with 1 . And 'B' can also dance with them, so B also has a number to dance with 1 . If they can both dance with 1, they can also dance with each other.

After Tom wrote this, Susan read it, nodded vigorously and said "that makes sense". It does make sense. They have here a justification for the claim that any two infinite sequences (without duplicates) can be placed in a one-to-one correspondence by combining the correspondences of each sequence with the natural numbers.

To summarise, all the students demonstrated understanding of the idea that if a robot can generate an infinite sequence then another robot can count the elements of that sequence. They were able to give arguments for why the rational numbers were dense since you can find an average between two numbers, and then the average between the result and the other number, and this process can be carried on ad infinitum. But the younger students were not consistent and seemed confused about how infinitely repeated decimal numbers fit into the picture again; this is hardly surprising.

The older English students decided to disagree about whether one can make a one-to-one correspondence between an interval of rational numbers and the entire set of rational numbers. The presence of negative numbers in only one set seemed to cause difficulties for some of them. When one child produced an insightful argument as to how to think about these mappings, it led an understanding by another one of the students.

\section{Constructing uncountable sets}

In the last activity, Counting all infinite sequences, students were acquainted with the fundamental theorem of Cantor that the set of real numbers cannot be placed in a one-to-one correspondence with the natural numbers, i.e. that there are different infinities.

They explored the topic by building robots that can generate a sequence missing from any purported sequence of all infinite sequences. This activity was designed to

- $\quad$ lead, in small steps, to an understanding of Cantor's famous diagonal proof that the real numbers are not countable; and

- $\quad$ reduce the likelihood that students will automatically conclude that all sets are countable.

Rather than focus on the set of real numbers, we chose to focus this activity around the set of infinite sequences. We expected infinite sequences to now be familiar objects to the students who have worked through the earlier activities. The Diagonal robot takes as input a sequence of different infinite sequences. It produces a sequence whose first element is the first element of the first sequence, whose second element is the second element of the second sequence, and so on. The students then give the output of the Diagonal robot to any robot that modifies each element (e.g. to the $A d d 1$ robot). Since each element differs in at least one location from the corresponding elements in all the incoming sequences, one can reason that the original sequence cannot have contained all infinite sequences. The robots constructed within this activity constitute an informal proof that the set of all infinite sequences is not countable. The students were then asked to reflect upon whether the resulting sequence could possibly be a term of the original input sequence:

Is it possible for the resulting sequence to be the same as one of the incoming sequences, i.e. to produce the same numbers in the same order?

In response Roger from the younger English group wrote:

No because Add One numbers can go on the input side but won't come [out] the same. 
Two boys in the second year group in England wrote:

No, when you want a number to be in a sequence it'll always be 1 more. Hard to explain.

While a girl (Susan) from that second year group wrote:

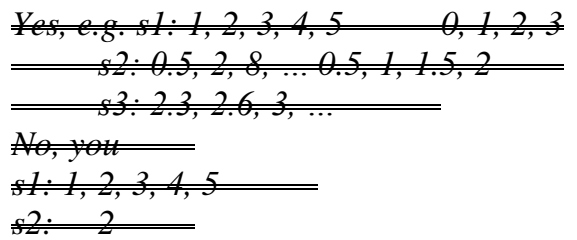

No, because for a number to be in both sequences it needs to be the same as itself +1 .

The worksheets show how all three students at first failed to see that they had constructed a sequence that differs in at least one location from every incoming sequence, and then they corrected themselves. Susan tried hard to construct an example of a sequence that would match the altered diagonal, as she repeatedly failed came to understand that there is no such sequence.

The first year boy, Roger, as he worked on all these questions, wrote several tables on the back of his folder (Figure 10). In the column labelled 'input', he drew 5 boxes each containing the beginning of different sequences. For example, the first contained 2, the second 5 and 9, and the third 10,15, and 3. He then filled in a column labelled 'output' with 2, 9, 3, and so on. He made a separate column labelled 'add one' and put in it 3,10, 4, and so on.

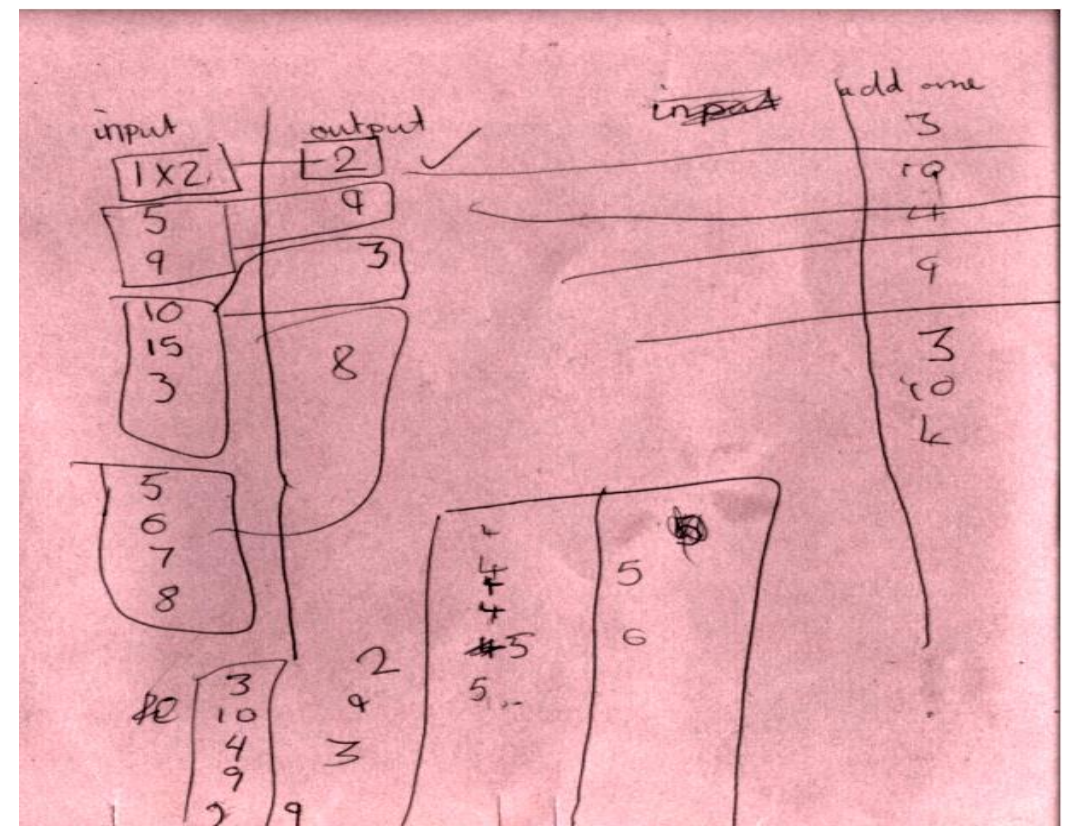

Figure 10 - An 11-year old boy working out the "diagonal" argument for uncountable sets For the last question, Susan from the second year wrote:

There can't be two sequences the same because we changed the diagonal sequence so the $x^{\text {th }}$ value (which was also the $x^{\text {th }}$ value of the sequence which was the same) has changed and is no longer the same.

At the end of this session this girl said "My brain hurts", and her classmate said he felt that way too. They later wrote the following web report in answer to the question: "Is the infinite sequence of all infinite sequences countable?"

It would be possible to imagine pairing each with a natural number, but you would never be able to physically do it and finish. If you have every sequence, you have to have an order. There are infinite orders, so you can only call this 'an' infinite sequence of all infinite sequences, not 'the' infinite sequence of all infinite sequences as there would be an infinite number of infinite sequences of all infinite sequences.

You cannot make an infinite sequence of all sequences because there is always a sequence that you haven't got - you can find this by taking the first term of the first 
sequence, the second term of the second sequence, the third term of the third sequence, the fourth term of the fourth sequence and the nth term of the nth sequence and changing it, e.g. by adding 1, or dividing by 670009. You will not however then have all the sequences because there will be lots of other sequence[s] like this.

They noticed that there are an infinite number of permutations of the sequence of all infinite sequences. Then they gave a good summary of Cantor's diagonalisation argument. They discussed the question of whether some of the sequences could themselves have been produced by a diagonal process and concluded it couldn't include them all.

\section{Concluding remarks}

We seem to have succeeded in our primary aim of enabling and guiding students to explore and reason about the cardinality of sets, as they build and use infinite computational processes. Moreover, as the students were led on a computational exploration of the cardinality of infinite sets, they often encountered other topics along the way. The Bulgarian students explored questions of how to compare the "growth" of sequences in a lengthy group report. Other "spin-off" topics include the convergence/divergence of infinite series, irrational numbers, and limits. Computer science topics arose frequently. Some of the students speculated about whether it is physically possible for a computation to go forever in a universe with a finite amount of matter or a finite future. Some of the students discussed whether there were sequences that couldn't be described and hence couldn't be programmed. Exploration of these ideas became relatively natural over the course of the work, natural in the sense that they could be expressed with the representations at hand - the representational tools of the WebLabs system.

In our sessions, we also observed that students developed a sense of how important a definition of a notion is in order to talk coherently about the concept. They were ready to modify their definitions in the process of the activity in accordance with their findings and discussions with the teachers. For comparing sequences we see different definitions coming from different students and groups and observe the process of modification, refinement and enrichment of those definitions.

The process of coming to a group consensus and publishing their findings and thoughts on a website led to surprisingly sophisticated discussions between the students. For example, as described above, the older students in both countries were comfortable with the idea that they could create multiple definitions of concepts such as infinite sequences and the relative size of infinite objects. They then successfully applied their definitions to different situations. They seemed to enjoy speculating about irrational numbers and non-describable or non-computable sequences. The younger English students were also inventing and applying their own definitions, for example, that $a$ sequence is larger if its elements are bigger. This is a perfectly good, even if mathematically non-standard, way of comparing infinite sequences. Unfortunately, it doesn't involve the one-to-one correspondences they had been exploring and creating in this activity sequence.

In conclusion, the students experienced a kind of mathematics very unusual in schools. They proposed their own definitions, explored them in the context of infinite objects, and reasoned about novel and paradoxical mathematical situations.

The students, despite being much younger than the usual age in which most of these concepts are introduced in schools, seem to have acquired intuitions and insights into the nature of infinity. They have

- seen how a proper subset can have a one-to-one correspondence with the whole set,

- understood how, for example, the set of every millionth positive integer has the same cardinality as the set of all rational numbers, and

- understood why no sequence can be constructed for the real numbers or the set of all infinite sequences.

The students experienced infinity in novel ways - as infinite computational processes that they designed, constructed and observed, and as tangible computational objects produced by their perpetual processes. And finally they became proficient at performing manipulations, applying operations, and doing comparisons of these infinite objects.

Even if it is difficult to assert how much the students have deepened their understanding of infinity it was worth designing activities that gave them a language to think about such an abstract concept. With that language, they constructed situated abstractions (Noss \& Hoyles, 1996) about infinity and they had useful discussions and collaborations. As Papert (1972) put it: "When 
mathematizing familiar processes is a fluent, natural and enjoyable activity, then is the time to talk about mathematizing mathematical structures, as in a good pure course on modern algebra". Thus, our main contribution was giving the students a representational infrastructure that changes fundamentally the way they explore, act with, discuss, and learn mathematical concepts and relationships. The infrastructure provides affordances and a substrate upon which we conjecture future learning could be built.

Our experience is that an understanding of the cardinality of infinite sets based upon perpetual computational processes can be acquired by students before they have the mathematical sophistication to approach these concepts via set theory. We believe a deep and thorough understanding of the topic can eventually be obtained by students who take both a classical set theory-oriented approach and the computational approach presented here.

What we can assert based on our WebLabs experience is that with carefully designed representational infrastructure, based on programming, even relatively young students can express deep ideas in quasi-formal ways, that they can enjoy mathematics and can be involved in deep mathematical discussions. And it was not in vain that appreciating mathematics was considered by Whitehead (as quoted by Hilton, 1991) to be one of the few pursuits in life (together with making music and the design of elegant and useful furniture) inherently worthwhile.

Today, five years later, it might be interesting to "hear" what some of these students remember from the WebLabs experiment:

Ivan: It is the infinity that I remember the most vividly from the ToonTalk classes. And the big numbers with which it was possible to show with great precision what is the behaviour of the sequence (at least with what I knew at the time). Today one can construct VERY BIG numbers in multiple of ways (Mathematica) but they are not as suitable and stimulating to work with as those in ToonTalk (not just to write "certain code") and the interface was very interesting for children.

Mitty: I got a very concrete image of the infinite sequences - when I would come across a sequence later at school I always imagined it as made by robots... Another thing I remember was that we put the numbers in couples in order to compare which set has more elements...

\section{References}

Dauben, J. W. (1990) Georg Cantor: His Mathematics and Philosophy of the Infinite. Princeton University Press: Princeton, NJ.

diSessa, A. (2000) Changing Minds. Computers, Learning and Literacy. Cambridge, MA: MIT Press.

Falk, R. and Ben-Lavy, S. (1989) 'How big is an infinite set? Exploration of children's ideas'. In Proceedings of the Thirteenth Annual Conference of the International Group for the Psychology of Mathematics Education, Vol. 1, pp. 252-259.

Fischbein, E., Tirosh, D. and Hess, P. (1979), 'The intuition of infinity', Educational Studies in Mathematics 10 , pp. 3-40.

Hilton, P. (1991) 'The Mathematical Component of a Good Education', Miscellanea Mathematica, Springer-Verlag, Berlin.

Kahn, K. (2001) 'Generalizing by Removing Detail', Communications of the ACM, 43(3), March 2000. An extended version is in Henry Lieberman, editor, Your Wish Is My Command: Programming by Example, Morgan Kaufmann, 2001.

Kaput, J., Noss, R. and Hoyles, C. (2002) "Developing New Notations for a Learnable Mathematics in the Computational Era", in: English, L. (ed.) Handbook of International Research in Mathematics Education, Lawrence Erlbaum, London, pp. 51-75. (Reprinted in Second Edition, 2008).

Lang, S. (1985) Math!: Encounters with High School Students. Springer-Verlag, New York Inc. Maor, E. (1987) To Infinity and Beyond: A Cultural History of the Infinite. Birkhäuser Boston, p. vii.

Monaghan, J. (2001) 'Young peoples' ideas of infinity', Educational Studies in Mathematics, 48, pp. $239-257$.

Mor, Y. \& Sendova, E. (2003) 'ToonTalking about mathematics', in I. Derzhanski, H. Dimitrova, S. Grozdev, E. Sendova (Eds) History and Education in Mathematics and Informatics, Attracting 
Talent to Science, Proceedings of the International Congress MASSEE 2003, September 15-21, Borovets, Bulgaria, pp. 36-43

Noss, R. \& Hoyles, C. (1996) Windows on Mathematical Meanings. Learning cultures and computers. Kluwer Academic Publishers: Dordrecht, Boston, London.

Papert, S. (1972) 'Teaching Children to be Mathematicians Versus Teaching About Mathematics'. International Journal of Mathematics Education and Science and Technology, 3, pp. 249-262.

Piaget, J. \& Garcia, R. (1989) Psychogenesis and the History of Science. Columbia University Press: New York.

Sacristán, A. I. (1997) Windows on the Infinite: Constructing Meanings in a Logo-based Microworld. PhD Dissertation, Institute of Education, University of London, UK.

Sacristán, A.I. \& Noss, R. (2008) 'Computational Construction as a Means to Coordinate Representations of Infinity.' International Journal of Computers for Mathematical Learning 13 (1), pp. 47-70.

Simon, M. A. and Tzur, R. (2004) 'Explicating the Role of Mathematical Tasks in Conceptual Learning: An Elaboration of the Hypothetical Learning Trajectory'. In Mathematical Thinking and Learning, 6(2), pp. 91-104.

Sussman, J. and Abelson, H. (1996) Structure and Interpretation of Computer Programs. MIT Press, Second edition.

Tall, D. O. (2001) A child thinking about infinity. In Journal of Mathematical Behavior, 20, pp. 719.

Tsamir, P. (2001) 'When 'The Same' is not perceived as such: The case of infinite sets', Educational Studies in Mathematics, 48, pp. 289-307.

Wilensky, U. \& Papert, S. (2010) "Restructurations: Reformulations of Knowledge Disciplines through new representational forms", In J. Clayson \& I. Kallas (Eds.), Proceedings of the Constructionism 2010 Conference. Paris, France.

\section{On-line Resources}

The software and activity sheets used in this study are available at no cost at http://www.toontalk.com/English/free.htm. The web reports posted by the students can be found

http://www.lkl.ac.uk/kscope/WebLabs/webreports/www.WebLabs.org.uk/wlplone /Reports/reports_index_html.html.

\section{Acknowledgements}

The WebLabs project was funded by Grant IST 2001-3220 of the Information Society Technologies Programme of the European Commission. We wish to thank the European Union for funding this project.

We are indebted to the Bulgarian institutions which provided excellent conditions for organizing the sessions on infinity: 21st Century Academy in Plovdiv, The Institute of Mathematics and Informatics at the Bulgarian Academy of Sciences, The Faculty of Mathematics and Informatics at Sofa University. Our deep gratitude to the Bulgarian coordinator, Dr Iliana Nikolova, and the teachers involved in the WebLabs project (George Gachev - in Sofia, and Liliana Moneva - in Plovdiv). And last, but not least - to the youngest participants in the project, the students, for their infinite creativity.

We are very grateful to the UK schools Theydon Bois Primary School and the Cherwell School in Oxford and their students who spent many hours using our software and learning materials to e

$\mathrm{X}$

$\mathrm{p}$

1

o

e 